Revue

Revue de l'histoire des religions

de Ihistoire des religions

3 | 2017

Varia

Théologie et érudition de la crise moderniste à Vatican II. Autour du Dictionnaire de théologie catholique, Textes réunis par Sylvio Hermann De FRANCESCHI

Limoges, Presses universitaires de Limoges, 2014

Jacques Le Brun

\title{
OpenEdition
}

Journals

Édition électronique

URL : http://journals.openedition.org/rhr/8809

DOI : $10.4000 /$ rhr.8809

ISSN : 2105-2573

Éditeur

Armand Colin

Édition imprimée

Date de publication : 1 septembre 2017

Pagination : $574-576$

ISBN : 978-2-200-93127-8

ISSN : 0035-1423

Référence électronique

Jacques Le Brun, "Théologie et érudition de la crise moderniste à Vatican II. Autour du Dictionnaire de théologie catholique, Textes réunis par Sylvio Hermann De FranceschI », Revue de l'histoire des religions [En ligne], 3 | 2017, mis en ligne le 01 septembre 2017, consulté le 07 janvier 2021. URL : http:// journals.openedition.org/rhr/8809; DOI : https://doi.org/10.4000/rhr.8809

Ce document a été généré automatiquement le 7 janvier 2021.

Tous droits réservés 


\section{Théologie et érudition de la crise moderniste à Vatican II. Autour du Dictionnaire de théologie catholique, Textes réunis par Sylvio Hermann De FRANCESCHI}

Limoges, Presses universitaires de Limoges, 2014

Jacques Le Brun

\section{RÉFÉRENCE}

Théologie et érudition de la crise moderniste à Vatican II. Autour du Dictionnaire de théologie catholique, Textes réunis par Sylvio Hermann De FRANCESCHI, Limoges, Presses universitaires de Limoges, 2014, 24 cm, 380 p., $28 €$, ISBN 978-2-84287-632-6.

1 Aujourd'hui encore nous nous servons du Dictionnaire de théologie catholique pour trouver des renseignements bio-bibliographiques ou des indications historiques ou théoriques sur telle notion d'usage en théologie; nous le faisons en n'ayant qu'une vague conscience d'utiliser comme indispensable instrument de travail une œuvre qui elle-même, de plusieurs points de vue, est un objet historique, sur lequel doit être tenu un discours historique. C'est là une analyse qui a été menée sur ce dictionnaire, sur ceux qui l'ont dirigé et ceux qui ont rédigé les articles, sur les questions abordées et celles qui ne l'ont pas été.

2 Car le DThC est d'abord historique par la durée qu'il a fallu pour aller d'Aaron à Zwinglianisme, de 1899 aux années 1970 (il faut aussi compter le temps de rédaction des tables, si utiles et qui jouent par les articles complémentaires le rôle d'un Supplément). Historique le DThC l'est par la succession des trois directeurs qui se sont dévoués (et sont morts) à la tâche, Alfred Vacant († 1901), Eugène Mangenot († 1922), Émile Amann 
(† 1948), auxquels il convient d'ajouter le maître d'œuvre des tables, Marie-Albert Michel († 1972). Chacun des directeurs avait son itinéraire institutionnel (ici on soulignera l'importance des professeurs du séminaire de Nancy), ses positions théologiques, ses compétences scientifiques, qui ne pouvaient pas ne pas influencer le choix des auteurs et des articles, l'orientation des positions théologiques exprimées au final dans l'article. L'œuvre est ainsi historique en quelque sorte par l'empilement des temporalités : celle du fait étudié (par exemple remontant à l'époque de saint Ambroise, au XVI ${ }^{e}$ ou au XIX ${ }^{e}$ siècle), puis la date de rédaction d'un article (qu'il soit écrit en 1900 ou en 1935 a son importance), celle de la publication avec son contexte historique et/ou théologique, enfin le temps de notre lecture un siècle après la rédaction ou la publication des articles, un temps où le développement de l'historiographie et la radicale mutation du contexte nous obligent à prendre des distances par rapport au texte des articles et à les analyser comme objet d'étude.

3 C'est avec la conscience de ces conditions que s'est tenu le colloque dont les actes sont publiés aujourd'hui avec à la fois des perspectives communes et des variantes tenant à la nature du sujet abordé dans chaque communication. Ouvrant le volume, nous lisons une très ample étude (p. 7-112, presque un livre !) par Sylvio Hermann De Franceschi, maitre d'œuvre du colloque et de cette publication, traçant l'histoire du dictionnaire, les faits et les orientations, l'évolution au fil des décennies, les problèmes de personnes, les débats historiques que révèle ou dissimule cette histoire. Par son exhaustivité, ses recherches de première main, la finesse des analyses, cette «Introduction ", avec son titre programmatique " Historiciser le travail des théologiens : du bon usage du DThC », constitue à la fois une entrée en matière et la synthèse qui donne sens au rassemblement des communications qui suivent.

4 À cette introduction, comme une série d'exemples significatifs, douze communications reprennent, de première main elles aussi, quelques aspects à partir desquels faire jouer le procès d'historicisation dont l'introduction traçait le programme. Il peut s'agir de monographies comme celle que consacre Étienne Fouilloux à l'abbé Amann, directeur de 1922 à sa mort en 1948. Plus souvent il s'agit d'un point de vue ou d'une question abordée dans une série d'articles du DThC: le traitement de l'Orient chrétien par Aurélien Girard, les rapports entre saint Ambroise et Théodose $\left(\mathrm{au} \mathrm{IV}^{\mathrm{e}}\right.$ siècle et jusqu'aux temps modernes) par Bertrand Lançon. Avec la contribution de Daniel-Odon Hurel c'est la présentation des bénédictins comme objets du dictionnaire et comme auteurs d'articles, avec celle d'Anne Massoni est analysée la représentation du clergé séculier, et avec celle d'Alain Rauwel sur les sacrements c'est l'opposition avec les "adversaires", protestants ou modernistes. Tous ces chapitres nous font saisir la permanence au $\mathrm{xx}^{\mathrm{e}}$ siècle des débats de l'âge classique que l'on croyait clos et qui se révèlent d'une brûlante actualité (au moins dans le milieu des rédacteurs ou des lecteurs du DThC !) : le gallicanisme (avec Frédéric Gabriel), la théologie morale et le probabilisme (Jean-Pascal Gay, montrant le rôle du P. Thomas Deman), la controverse sur la grâce entre thomistes et molinistes qui semble ne pas avoir évolué depuis les congrégations De Auxiliis au début du xvII ${ }^{\mathrm{e}}$ siècle (Sylvio Hermann De Franceschi), le jugement porté sur les Lumières (Caroline Chopelin-Blanc), le libéralisme (Sylvain Milbach). Le dernier chapitre constitue une parfaite conclusion: Christian Sorrel montre comment les débats et la crise modernistes "enveloppent l'histoire du DThC » et font planer leur ombre sur un dictionnaire qui s'ouvre sur les prodromes de la crise et se ferme aux lendemains de l'encyclique Humani generis (1950). 
De ces travaux se dégagent quelques grandes orientations : nous savions que tous les dictionnaires que nous utilisons chaque jour (Altenstaig, Moréri, Bayle, Chaufepié, les encyclopédies Migne, etc.) étaient "datés », mais il était intéressant de voir comment une œuvre que nous pouvions croire plus près de nous en était peut-être aussi éloignée. Le DThC regarde en effet vers le passé ; devant ces articles, le lecteur d'aujourd'hui découvre, selon l'excellente formule d'Alain Rauwel, "un monde qui se ferme ", une "théologie contra». Ce monde, où une incontestable érudition s'accompagne d'une étonnante cécité aux mutations de la société et de la pensée au $\mathrm{xx}^{\mathrm{e}}$ siècle, c'est celui de la «science catholique » que plusieurs travaux (rappelons ceux du regretté François Laplanche et ceux de Claude Langlois) nous ont fait redécouvrir, montrant, ce que le DThC montre aussi, l'obsession des Français pour une "science allemande » (Mommsen, le CSEL, etc.), objet d'envie et de répulsion. Le DThC c'est aussi, en plein $\mathrm{xx}^{\mathrm{e}}$ siècle, un espace de recherches dont sont étonnamment absentes les sciences, la philosophie et la psychanalyse, comme si la théologie pouvait s'absenter du monde dans lequel elle déploie ses certitudes éternelles.

\section{AUTEURS}

\section{JACQUES LE BRUN}

École Pratique des Hautes Études, Paris. 